\title{
Magnetars and Gamma Ray Bursts
}

\author{
Niccolò Bucciantini \\ INAF, Osservatorio Astrofisico di Arcetri, L.go Fermi 5, 50125, Firenze, Italia \\ email: niccolo@arcetri.astro.it
}

\begin{abstract}
In the last few years, evidences for a long-lived and sustained engine in Gamma Ray Bursts (GRBs) have increased the attention to the so called millisecond-magnetar model, as a competitive alternative to the standard collapsar scenario. I will review here the key aspects of the millisecond magnetar model for Long Duration Gamma Ray Bursts (LGRBs). I will briefly describe what constraints present observations put on any engine model, both in terms of energetics, outflow properties, and the relation with the associated Supernova (SN). For each of these I will show how the millisecond magnetar model satisfies the requirements, what are the limits of the model, how can it be further tested, and what observations might be used to discriminate against it. I will also discuss numerical results that show the importance of the confinement by the progenitor star in explaining the formation of a collimated outflow, how a detailed model for the evolution of the central engine can be built, and show that a wide variety of explosive events can be explained by different magnetar parameters. I will conclude with a suggestion that magnetars might be at the origin of the Extended Emission (EE) observed in a significant fraction of Short GRBs.
\end{abstract}

Keywords. (magnetohydrodynamics:) MHD, stars: neutron, stars: magnetic fields, stars: winds, outflows, (stars:) supernovae: general, gamma rays: bursts

\section{Introduction}

The key idea behind the so called magnetar model for LGRBs (Usov 1992,Thompson 1994, Wheeler et al. 2000, Thompson et al. 2004) assumes that the collapse of the core of a massive star at the end of its life leads to a rapidly rotating proto-neutron star (NS; period $\sim 1 \mathrm{~ms})$, with a strong surface magnetic field $\left(B \geqslant 10^{15} \mathrm{G}\right)$. There are reasons to believe that these two properties might be related by dynamo processes (Thompson \& Duncan 1995, Thompson \& Duncan 1996), even if this might be debated and requires progenitors with rapidly rotating cores. The maximum energy that can be stored in a rotating NS is $\sim 2 \times 10^{52} \mathrm{erg}$, and the typical timescale over which this energy can be extracted and delivered to the surrounding medium is $\sim 100$ s for a magnetic field $\sim 3 \times 10^{15} \mathrm{G}$. These energies and timescales are compatible with almost every LGRB observed. Moreover, the formation of a proto-NS is fundamental for a successful supernova explosion (NSs are known to produce relativistic outflows; Bucciantini 2008), and there are evidences that massive stars might not necessarily end their life forming Black Holes (Muno et al. 2006, Belczynski \& Taam 2008, Gaensler et al. 2005, Wachter et al. 2008, DeLaney et al. 2006 , Vink 2008, Morton et al. 2007). Indeed the rotational energy available in a millisecond proto-NS is more than sufficient to unbind the envelope of even a 40-60 $M_{\odot}$ star (Metzger et al. 2011), and, for a strong magnetic field mass losses can outpace accretion (Dessart et al. 2008) and the wind might halt the fallback of marginally bound ejecta (Bucciantini et al. 2009) 


\section{Energetics and timescales for proto-NS winds}

Once a proto-NS is formed it will cool via neutrino emission in a typical KelvinHelmholtz timescale $t_{K H} \sim 10-100 \mathrm{~s}$ (Pons et al. 1999). Neutrinos deposit heat in a neutrinosphere and at about 500-800 ms after core bounce the density surrounding the proto-NS can drop to the point where a neutrino driven wind develops (Arcones et al. 2007, Thompson et al. 2001). For proto-NS with pulsar like magnetic fields, this wind carries negligible energy (Thompson et al. 2001). However, for magnetar like magnetic fields, the wind is magnetocentrifugally accelerated and far more energetic (Thompson et al. 2004). As the neutrino luminosity decreases, and the mass loss rate drops, the wind becomes progressively more magnetized eventually reaching relativistic speeds (Thompson et al. 2004, Bucciantini et al. 2006).

In a recent paper, Metzger et al. (2011) have developed a full model for the spin-down evolution of a proto-NS, taking into account the neutrino cooling, the magnetic torque by the wind, and the possible inclination of the magnetic axis with respect to the spin axis. The model can be used to estimate the energy flux in the wind and its magnetization parameter $\sigma$ which is defined as the ratio of Poynting flux over kinetic energy flux, and can be thought of as a proxy for the maximum achievable Lorentz factor.

Four phases can be distinguished in the overall evolution (Metzger et al. 2011):

- An early non relativistic phase, that lasts $\sim 1 \mathrm{~s}$ after bounce, when the proto-NS is still hot and contracting, the wind is magnetized, but with typical terminal speeds $\sim 0.1 c$.

- An intermediate mildly relativistic phase that lasts $\sim 10$ s after bounce. The protoNS relaxes to a radius $\sim 20 \mathrm{~km}$ and begins to spin-down, the wind mass loss rate drops, and $\sigma$ increases from values of $\sim 1$ to $\sim 10$. The wind is now relativistic but still confined inside the progenitor.

- The GRB phase, after the wind breaks out of the progenitor and starts to accelerate in the circumstellar medium. Due to the decreased neutrino luminosity and related mass loss rate, the magnetization rapidly increases to values of $\sigma \sim 10^{2}-10^{3}$, while energy losses are still high $\left(\sim 10^{49} \mathrm{erg} \mathrm{s}^{-1}\right)$. This phase lasts for about $100 \mathrm{~s}$, when the neutrino luminosity drops sharply below the threshold to drive a baryon loaded wind.

- A late activity phase that begins once the baryon loaded wind ceases, to be replaced by a leptonic wind once the density in the magnetosphere drops below the threshold for pair production. The wind luminosity is smaller $\left(\sim 10^{48} \mathrm{erg} \mathrm{s}^{-1}\right)$ but the typical spin-down time is now longer $(\sim 100 \mathrm{~s})$, as expected to explain some aspects of the late activity observed in some LGRBs.

We want to emphasize here that energy losses are due to a magnetized stellar wind, analogous to the Solar wind and other stellar winds, and are not due to magnetic dipole radiation. Interestingly for $\sigma \gg 1$ the two have the same value, but in the early and mildly relativistic phases the energy losses in the wind can exceed even by a factor of 10 what can be estimated based on dipole radiation alone.

\section{Collimation and acceleration of the outflow}

As opposed to accretion disks around black holes that are known to power collimated outflow in the form of relativistic jets, magnetars are supposed to produce essentially spherical outflows. Relativistic outflows cannot self collimate (Lyubarsky \& Eichler 2001), and at large distances from the Light Cylinder the wind structure should closely follow the split monopole solution (Bucciantini et al. 2006) where most of the energy flux is in the equatorial region. Moreover in monopolar relativistic outflows, the terminal Lorentz 
factor can only be as high as $\sigma^{1 / 3}$ (Arons 2002). This is called the $\sigma$ problem. It is evident that the GRB outflow that is observed cannot coincide with the the steady state spherical wind emerging directly from the proto-NS magnetosphere.

We know that as the outflow emerges from the interior of the host star it collimates into a jet that punches through the stellar envelope, creating a channel where material coming from the central engine can flow (e.g., Matzner 2003). Afterglow observations (jet breaks; Rhoads 1999) and GRB energetics (comparison of the total energy derived from late radio afterglow observations with respect to the prompt emission) confirm that a collimated flow is present.

Simple energy considerations demonstrate that the surrounding stellar envelope provides an efficient confining medium even for a very energetic proto-magnetar wind. It is indeed the interaction with the progenitor star that provides the collimating agent to channel the spherical magnetar wind into a polar jet. By analogy to bipolar wind bubbles (Königl \& Granot 2002, Begelman \& Li 1992), the interaction of the wind from the spinning-down magnetar with the surrounding star could facilitate collimation. It has been shown, under different assumptions, that once the interaction with the surrounding progenitor is taken into account, this can in fact occur (Bucciantini et al. 2007, Bucciantini et al. 2008, Komissarov \& Barkov 2007, Bucciantini et al. 2009).

The physical picture is based on an analogy to the case of pulsar wind nebulae (PWNe; Komissarov \& Lyubarsky 2004, Del Zanna et al. 2004): the magnetar wind is confined by the surrounding (exploding) stellar envelope, as a result a magnetar wind nebula (MWN) forms where the wind magnetic field is compressed. If the toroidal magnetic field in the bubble is sufficiently strong, due to the tube of toothpaste effect the bubble expands primarily in the polar direction while a negligible amount of energy is transferred to the $\mathrm{SN}$ envelope.

The issue of collimation is strictly related to the problem of the acceleration because deviations from the strict monopole geometry can substantially enhance the terminal Lorentz factor, as well as time dependent effects. In the millisecond magnetar, the collimation of the outflow and the formation of a wind nebula becomes a key features if one wants to build a GRB engine. A key assumption here is that magnetic energy can be efficiently dissipated/converted into kinetic energy (Arons 2002, Lyubarsky \& Kirk 2001 , Kirk \& Skjæraasen 2003). This can happen in various ways. For an oblique rotator, the striped magnetic field might reconnect and dissipate at the wind termination shock of the nebula as it slows down (Lyubarsky 2005). It is not unlikely that instabilities and dissipation might be at work inside the nebula itself or in the jet (Begelman \& Li 1992, Moll et al. 2008). Modulation of the outflow by the confining walls of the channel formed inside the progenitor might enhance magnetic to kinetic energy conversion (Granot et al. 2011).

Results show that the opening angle of the jet, as it punches trough the star and later emerges into the circumstellar medium, is of order $5-10^{\circ}$ and appears to be independent of the dissipative properties of the magnetar wind, as long as the magnetization inside the MWN reaches equipartition. It is also shown that the outflow can accelerate rapidly as soon as it emerges from the progenitor star.

\section{SN association and late activity}

It is now well established that long-duration GRBs are associated with core-collapse $\mathrm{SNe}$, in particular with the subclass of SNe Ic-BL (BL = broad line; Woosley \& Bloom 2006, Della Valle 2006, Zhang 2007). Interestingly, the converse is not true (Soderberg 2006, Woosley \& Bloom 2006), and the search for orphan afterglows shows that within 
a high confidence level the hypothesis that every broad-lined SN harbors a GRB can be ruled out. Moreover, even if the SNe associated with long-duration GRBs tend to be more luminous than the average sample, they are not particularly unusual among the class of BL SN in terms of their energies, photospheric velocities, and Ni masses.

It can be debated if this class of core-collapse SNe that are unusually energetic, asymmetric (as revealed by spectra-polarimetry), and produce significant amounts of $\mathrm{Ni}$ are powered by a diverse central engine (a failed GRB), or if the GRB engine is only a possible outcome of the conditions leading to those supernovae: are hypernovae due to a GRB-like engine, or vice-versa? Higher energies, axisymmetry, and Nickel production are the three aspects that must be considered in the GRB-SN association.

In the magnetar model nearly all of the spindown energy of the neutron star escapes in the polar channel (Komissarov \& Barkov 2007, Bucciantini et al. 2009). There is very little coupling between the exploding star and the GRB engine. This seems to apply both to the low (dissipative) and high (non dissipative) $\sigma$ limits. The interesting implication of this result is that a proto-magnetar powering a GRB is unlikely to contribute significantly to energizing the SN shock as a whole (although it clearly does so in the polar region), at least on timescales $\gtrsim 1$ sec after core bounce. This is a key property of magnetized outflows and in principle it is not specific to a particular central engine. Specifically, we suspect that the same results will apply also to winds from accretion disks (Proga et al. 2003) that will likely escape via the polar channel rather than transferring energy to the SN shock as has been previously hypothesized (Arons 2003).

Given the relatively on-axis viewing angle of observed GRBs, high velocity ejecta might be observed; high velocity $\mathrm{O}$ and $\mathrm{Ne}$ can also be produced by the jet blowing out stellar material that had been processed during stellar evolution (Mazzali et al. 2006). A jet might lead to unique observable signatures in the ejecta at late times (as may be the case for Cas A; Wheeler et al. 2008).

A separate issue relates magnetar engines and the production of excess ${ }^{56} \mathrm{Ni}$ that are observed in hypernovae. It has been shown that the temperature at which explosive nucleosynthesis of ${ }^{56} \mathrm{Ni}$ happens $\left(\gtrsim 5 \times 10^{9} \mathrm{~K}\right.$; Woosley et al. 2002) is not attained even at relatively early times. This happens because, by the time the jet-plume emerges outside the SN shock, the density of the progenitor into which it propagates is $\sim 10^{4-5} \mathrm{~g} \mathrm{~cm}^{-3}$. At these densities $\mathrm{Ni}$ production requires a shock moving at nearly the speed of light, significantly faster than what can be achieved at these early times (Komissarov \& Barkov 2007, Bucciantini et al. 2009, Metzger et al. 2007). However, $\sim 10^{-2} M_{\odot}$ of high speed $(v \simeq 0.1-0.2 c)$ Ne and $\mathrm{O}$ can be created because these have lower threshold temperatures for successful explosive nucleosynthesis.

As a side point, the specific angular momentum required for a millisecond magnetar engine is $J \simeq 3 \times 10^{15} R_{10}^{2} P_{1}^{-1} \mathrm{~cm}^{2} \mathrm{~s}^{-1}$ (Thompson et al. 2004), which is about a factor of five smaller than what is required for the formation of an accretion disk for the blackhole accretion-disk model (MacFadyen \& Woosley 1999). This implies that if the core has enough angular momentum to power a collapsar engine, then it has enough to create a millisecond magnetar.

The millisecond magnetar model for LGRBs is particularly interesting in view of the so called late activity. Late activity manifests itself in the afterglow up to $10^{4-5}$ seconds after the prompt emission (Campana et al. 2005, Vaughan et al. 2006, Cusumano et al. 2006 , Nousek et al. 2006, O'Brien et al. 2006, Willingale et al. 2007), either as a shallow decay or plateau of the light curve, or with the presence of flares (Burrows et al. 2005, Falcone \& The Swift Xrt Team 2006 , Chincarini et al. 2007 ). Late activity requires a persistent engine at times much longer than the typical duration of the prompt emission to provide continuous injection of energy. 
In the millisecond magnetar model, late time injection can take the form of a leptonic wind, not dissimilar to standard pulsars. The amount of energy that can be released in this scenario, even if smaller than the prompt emission, can produce the shallow decay phase that is observed (Yu \& Dai 2007, Metzger et al. 2011).

More intriguing is the presence of flares that can carry a substantial fraction of energy compared to the prompt emission. A possibility is that these flares are the signature of magnetic readjustments, within the proto-magnetar that give rise to bursting activity not dissimilar to what is observed during giant bursts in SGRs (Thompson \& Duncan 1995, Thompson \& Duncan 1996, Woods 2004, Mereghetti 2008). The magnetic energy stored in canonical magnetars is smaller than the rotational energy required for a GRB engine, however the internal magnetic field might be much higher than the surface value (Braithwaite \& Spruit 2006, Braithwaite 2008).

\section{Validating the model for LGRBs}

As shown before, the magnetar model can reproduce many aspects of the observed phenomenology in LGRBs. It is interesting to evaluate if and how one can distinguish a magnetar from a different engine (i.e. a Black Hole). Unfortunately, the dynamical properties of magnetized outflows, once the value of Lorentz factor and of $\sigma$ are set, are largely independent on the conditions at injection. A more promising discriminant might be the composition: in particular within the magnetar model one expects a transition from a baryon loaded wind to a leptonic dominated outflow at $\sim 100 \mathrm{sec}$ after bounce.

Perhaps the bigger discriminant is the available energy. A magnetar can store at most a few times $10^{52}$ erg of energy. The detection of a GRB with higher total energy, could rule out a magnetar as its engine. Determining with accuracy the total energy of a GRB is non trivial. The prompt emission must be corrected for beaming (Cenko et al. 2010) and off-axis effects (van Eerten et al. 2010), while the late radio emission is often assumed to originate from a Sedov phase to be converted into a kinetic energy (Shivvers \& Berger 2011). There is a small set of very energetic GRBs (Cenko et al. 2010) that are marginally compatible with a magnetar engine. However for the vast majority, and for those for which we have good data, the inferred energies are always a few $10^{51}$ ergs (Shivvers \& Berger 2011).

There is also a set of GRBs with a long prompt emission characterized by several events lasting $\sim 100 \mathrm{sec}$ and separated by quiescence periods of about $200-400 \mathrm{sec}$. Unlike for a BH scenario where one might invoke bursty mass accretion, the magnetar spin-down is smooth. However, the gamma-ray luminosity might not be a good tracer of the energy injection, depending on the efficiency of particle acceleration in the outflow. In the recent paper by Metzger et al. (2011) it was shown that several expected correlations, like the Amati relation, can be recovered in the magnetar model assuming magnetic dissipation to be at the origin of the radiation mechanism.

\section{Short GRBs with extended emission}

The standard LGRB/SGRB dichotomy has recently been challenged by several 'hybrid' events that conform to neither class (e.g. Zhang 2007; Bloom et al. 2008). All together $\sim 1 / 4$ of Swift SGRBs are accompanied by extended X-ray emission lasting for $\sim 10-$ $100 \mathrm{~s}$ with a fluence approximately greater than that of the GRB itself (see Norris \& Bonnell 2006 and Perley et al. 2009 for a compilation of events). The hybrid nature and common properties of these events ('Short GRB' $+\sim 100 \mathrm{~s}$ X-ray tail) have motivated the introduction of a new subclass: Short GRBs with Extended Emission (SGRBEEs). 
It was moreover recently discovered that some SGRBs are followed by an X-ray 'plateau' ending in a very sharp break (GRB 980515; Rowlinson et al. 2010; Troja et al. 2008; Lyons et al. 2010), which is difficult to explain by circumstellar interaction alone. Although the connection of this event to SGRBEEs is unclear, it nevertheless provides additional evidence that the central engine is active at late times. The long duration and high fluence of the extended emission of SGRBEEs poses a serious challenge to the NS merger scenario, because in this model both the prompt and extended emission are necessarily powered by black hole accretion. It is in particular difficult to understand how such a high accretion rate is maintained at very late times. Metzger et al. 2008 recently proposed that SGRBEEs result from the birth of a rapidly spinning proto-magnetar, created by a NS-NS merger or the AIC of a WD. In this model the short GRB is powered by the accretion of the initial torus (similar to standard NS merger models), but the EE is powered by a relativistic wind from the proto-magnetar at later times, after the disk is disrupted. Although a NS remnant is guaranteed in the case of AIC, the merger of a double NS binary could also leave a stable NS remnant. The interaction of the relativistic proto-magnetar wind with the expanding ejecta was investigated by Bucciantini et al. (2012), with a focus on the confining role of the ejecta and its dependence on the wind power and on the ejecta mass and density profile. The model thus predicts a class of events for which the EE is observable with no associated short GRB. These may appear as long-duration GRBs or X-Ray Flashes unaccompanied by a bright supernova and not solely associated with massive star formation, which may be detected by future all-sky X-ray survey missions.

\section{References}

Arcones, A., Janka, H.-T., \& Scheck, L. 2007, A\& A, 467, 1227

Arons, J. 2002, Neutron Stars in Supernova Remnants, ASPC, 271, 71

Arons, J. 2003, ApJ, 589, 871

Belczynski, K. \& Taam, R. E. 2008, ApJ, 685, 400

Begelman, M. C. \& Li, Z. 1992, ApJ, 397, 187

Bloom, J. S., Butler, N. R., \& Perley, D. A. 2008, American Institute of Physics Conference Series, 1000, 11

Braithwaite, J. \& Spruit, H. C. 2006, A\&A, 450, 1097

Braithwaite, J. 2008, MNRAS, 386, 1947

Bucciantini, N., Thompson, T. A., Arons, J., Quataert, E., \& Del Zanna, L. 2006, MNRAS, 368, 1717

Bucciantini, N., Quataert, E., Arons, J., Metzger, B. D., \& Thompson, T. A. 2007, MNRAS, 380,1541

Bucciantini, N., Quataert, E., Arons, J., Metzger, B. D., \& Thompson, T. A. 2008, MNRAS, $383, \mathrm{~L} 25$

Bucciantini, N. 2008, 40 Years of Pulsars: Millisecond Pulsars, Magnetars and More, 983, 186

Bucciantini, N., Quataert, E., Metzger, B. D., Thompson, T. A., Arons, J., \& Del Zanna, L. 2009, MNRAS, 396, 2038

Bucciantini, N., Metzger, B. D., Thompson, T. A., \& Quataert, E. 2012, MNRAS, 419, 1537

Burrows, D. N., et al. 2005, Science, 309, 1833

Campana, S., et al. 2005, ApJL, 625, L23

Cenko, S. B., Frail, D. A., Harrison, F. A., et al. 2010, ApJ, 711, 641

Chincarini, G., et al. 2007, ApJ, 671, 1903

Cusumano, G., et al. 2006, ApJ, 639, 316

DeLaney, T., Gaensler, B. M., Arons, J., \& Pivovaroff, M. J. 2006, ApJ, 640, 929

Della Valle, M. 2006, Chinese Journal of Astronomy and Astrophysics Supplement, 6, 010000

Del Zanna, L., Amato, E., \& Bucciantini, N. 2004, A\&A, 421, 1063 
Dessart, L., Burrows, A., Livne, E., \& Ott, C. D. 2008, ApJL, 673, L43

Falcone, A. D., The Swift Xrt Team 2006, AIP, Gamma-Ray Bursts in the Swift Era, 836, 386

Gaensler, B. M., McClure-Griffiths, N. M., Oey, M. S., Haverkorn, M., Dickey, J. M., \& Green, A. J. 2005, ApJL, 620, L95

Granot, J., Komissarov, S. S., \& Spitkovsky, A. 2011, MNRAS, 411, 1323

Kirk, J. G. \& Skjæraasen, O. 2003, ApJ, 591, 366

Komissarov, S. S. \& Lyubarsky, Y. E. 2004, MNRAS, 349, 779

Komissarov, S. S. \& Barkov, M. V. 2007, MNRAS, 382, 1029

Königl, A. \& Granot, J. 2002, ApJ, 574, 134

Lyons, N., O'Brien, P. T., Zhang, B., et al. 2010, MNRAS, 402, 705

Lyubarsky, Y. \& Kirk, J. G. 2001, ApJ, 547, 437

Lyubarsky, Y. E. \& Eichler, D. 2001, ApJ, 562, 494

Lyubarsky, Y. 2005, Advances in Space Research, 35, 1112

MacFadyen, A. I. \& Woosley, S. E. 1999, ApJ, 524, 262

Matzner, C. D. 2003, MNRAS, 345, 575

Mazzali, P. A., et al. 2006, ApJ, 645, 1323

Mereghetti, S. 2008, The Astronomy and Astrophysics Review, 15, 225

Metzger, B. D., Thompson, T. A., \& Quataert, E. 2007, ApJ, 659, 561

Metzger, B. D., Quataert, E., \& Thompson, T. A. 2008, MNRAS, 385, 1455

Metzger, B. D., Giannios, D., Thompson, T. A., Bucciantini, N., \& Quataert, E. 2011, MNRAS, 413, 2031

Moll, R., Spruit, H. C., \& Obergaulinger, M. 2008, A\&A, 492, 621

Morton, T. D., Slane, P., Borkowski, K. J., Reynolds, S. P., Helfand, D. J., Gaensler, B. M., \& Hughes, J. P. 2007, ApJ, 667, 219

Muno, M. P., et al. 2006, ApJL, 636, L41

Norris, J. P. \& Bonnell, J. T. 2006, ApJ, 643, 266

Nousek, J. A., et al. 2006, ApJ, 642, 389

O'Brien, P. T., et al. 2006, ApJ, 647, 1213

Panov, I. V. \& Janka, H.-T. 2009, A\&SA, 494, 829

Perley, D. A., Metzger, B. D., Granot, J., et al. 2009, ApJ, 696, 1871

Piran, T. 1999, Physics Reports, 314, 575

Pons, J. A., Reddy, S., Prakash, M., Lattimer, J. M., \& Miralles, J. A. 1999, ApJ, 513, 780

Proga, D., MacFadyen, A. I., Armitage, P. J., \& Begelman, M. C. 2003, ApJL, 599, L5

Rhoads, J. E. 1999, ApJ, 525, 737

Rowlinson, A., O'Brien, P. T., Tanvir, N. R., et al. 2010, MNRAS, 409, 531

Shivvers, I. \& Berger, E. 2011, ApJ, 734, 58

Soderberg, A. M. 2006, Gamma-Ray Bursts in the Swift Era, AIP, 836, 380

Thompson, C. 1994, MNRAS, 270, 480

Thompson, C. \& Duncan, R. C. 1995, MNRAS, 275, 255

Thompson, C. \& Duncan, R. C. 1996, ApJ, 473, 322

Thompson, T. A., Burrows, A., \& Meyer, B. S. 2001, ApJ, 562, 887

Thompson, T. A. 2003, ApJL, 585, L33

Thompson, T. A., Chang, P., \& Quataert, E. 2004, ApJ, 611, 380

Troja, E., King, A. R., O'Brien, P. T., Lyons, N., \& Cusumano, G. 2008, MNRAS, 385, L10

Usov, V. V. 1992, Nature, 357, 472

van Eerten, H., Zhang, W., \& MacFadyen, A. 2010, ApJ, 722, 235

Vaughan, S., et al. 2006, ApJ, 638, 920

Vink, J. 2008, Advances in Space Research, 41, 503

Wachter, S., Ramirez-Ruiz, E., Dwarkadas, V. V., Kouveliotou, C., Granot, J., Patel, S. K., \& Figer, D. 2008, Nature, 453, 626

Willingale, R., et al. 2007, ApJ, 662, 1093

Wheeler, J. C., Yi, I., Höflich, P., \& Wang, L. 2000, ApJ, 537, 810

Wheeler, J. C., Maund, J. R., \& Couch, S. M. 2008, ApJ, 677, 1091

Woods, P. M. 2004, Advances in Space Research, 33, 630 
Woosley, S. E., Heger, A., \& Weaver, T. A. 2002, Reviews of Modern Physics, 74, 1015

Woosley, S. E. \& Bloom, J. S. 2006, ARA\&A, 44, 507

Yu, Y. W. \& Dai, Z. G. 2007, A\&A, 470, 119

Zhang, B. 2007, Chinese Journal of Astronomy and Astrophysics, 7, 1

\section{Discussion}

B. ZHANG: So you don't believe the magnetar model for Superluminous SNe

N. BuCCiAntini: A millisecond magnetar might have enough energy to power a Superluminous SN, but this energy, extrtacted by a magnetized wind, is only weakly coupled with the SN shock. I do not think that a magnetar can energize a SN shock, early enough to drive the nucleosynthesys of $\sim 0.5 M_{\odot}$ of ${ }^{56} \mathrm{Ni}$.

B. ZHANG: For NS-NS making a magnetar have you considered how a supermassive magnetar form? What kind of NS equation of state is needed.

N. BuCCIANTINI: For NS-NS merger resulting in a long lived magnetar, one needs peculiar conditions: two low-mass NSs must be involved; a few tenths $M_{\odot}$ must be lost either by strong neutrino driven winds or during the merger itself; the EoS must be particularly stiff. The existence of a $2 M_{\odot}$ NS suggests that the EoS might allow for massive magnetars.

S. MoISEENKO: In our simulations of magneto-rotational supernovae explosion we found that magnetic field can reach the values $10^{14}-10^{15} \mathrm{G}$, but it is chaotic magnetic field which can be reduced in a short time due to reconnection. How to make a neutron star with so strong magnetic field?

N. BuCCIANTINI: It is true that MRI, and other instabilities migh enhance strongly the magnetic field, but this happens at small scales, and the resulting field is mostly chaotic and tends to dissipate rapidly. One of the ideas behind the origin of the strong magnetar magnetic field, is that dynamo processes are at work. The key idea here is that a mean field dynamo operates. Investigating the possibility of mean field dynamo requires a full 3D geometry, with enough resolution to properly sample the parameter space in term of viscosity and resistivity. 2D simulation will all be subject to Cowling antidynamo theorem, so they can never lead to large scale fields. To my knowledge investigation of MHD Supernovae in the full 3D regime is very demanding and quite limited. We do observe magnetar, so nature must find a way to produce them. 